\title{
Ventricular tachycardia in normal heart
}

\author{
Dr. B. Rawat MD, $D M^{*}$, Dr. Sabita Malla $M D^{* *}$
}

Ventricular tachycardia is usually thought occurring only in abnormal heart. But it can also occur in apparently normal heart.

Cardiovascular evaluation is normal except for the arrhythmia. Monomorphic ventricular tachycardia is not always induced at electrophysiological study. The natural history is incompletely known, but recurrences are not uncommon. Antiarrhythmic drugs, radio frequency catheter ablation and implantable defibrillators are useful therapeutic choices. Prognosis is good even though occasional cases of sudden deaths have been reported.

Ventricular tachycardia arises distal to the bifurcation of the His bundle, in the specialized conduction system, in ventricular muscle, or in combinations of both tissue types. The mechanisms include disorders of impulse formation and conduction.

The electrocariographic diagnosis of VT is suggested by the occurrence of a series of three or more consecutive, bizarrely shaped premature ventricular complexes whose duration exceeds $120 \mathrm{msec}$, with the ST-T vector pointing opposite to the major QRS deflection. The R-R interval can be exceedingly regular or can vary. Patients can have VT with multiple morphologies originating at the same or closely adjacent sites, probably with different exit paths. Depending on the particular type of ventricular tachycardia, the rates range from 70 to 250 beats/min, and the onset can be paroxysmal (sudden) or nonparoxysmal. QRS contours during the VT can be unchanging (uniform, monomorphic), can vary randomly (multiform, polymorphic, or pleomorphic), can vary in amore or less repetitive manner (torsades de pointes), can vary in alternate complexes (bidirectional VT), or vary ina stable but changing contour (RBB contour changing to LBB contour). VT can be sustained, defined as lasting longer than $30 \mathrm{sec}$ or requiring termination because of hemodynamic collapse, or nonsustained, when it stops spontaneously in less than $30 \mathrm{sec}$.

$\mathrm{t}$ 


\section{Epidemiology and General Features}

Prevalence of VT in normal heart is $10-15 \%$ of all VTs. Mean age is 35 years. Even though the VT is well tolerated it is not so benign. Resting ECG is normal with no VLPs. During VT, QRS width is narrower and usually amplitude is higher. As compared to secondary VTs, it has a favorable prognosis. Long term treatment as RF catheter ablation is necessary if there are recurrent symptomatic episodes,

Which gives excellent results.

\section{Etiology}

VT in patients with apparently normal hearts may represent a true primary electrical disease but it may also be the first manifestation of a cardiomyopathy. Diseases with minimal anatomical abnormalities or in early stages may yet precipitate VT eg. myocarditis, idiopathic HCM MVP, idiopathic DCM, long QT syndrome, ARVD, sarcoidosis, amyloidosis, metabolic or electrolytes disturbances, proarrhythmic drug effects, CAD, coronary anomalies etc.

\section{Diagnosis}

After excluding a diseased heart by clinical assessment, echo and cardiac catheterization the diagnosis of VT in a normal heart is considered. Sometimes endomyocardial biopsy and electrophysiological parameters may be useful.

\section{There are $\mathbf{4}$ types of VT in normal heart:}

1. Idiopathic right ventricular tachycardia

2. Idiopathic left ventricular tachycardia

3. Recurrent monomorphic ventricular tachycardia

4. Bundle branch ventricular tachycardia

\section{Idiopathic Right Ventricular Tachycardia}

This is the commonest type of VT in a normal heart. The salient features here are LBBB with RAD (origin:RVOT) in the ECG, critical rate dependant window of sinus rate and episodes of VT frequently related to physical activities. 


\section{Fig. 1: IDIOPATHIC RIGHT VENTRICULAR TACHYCARDIA}
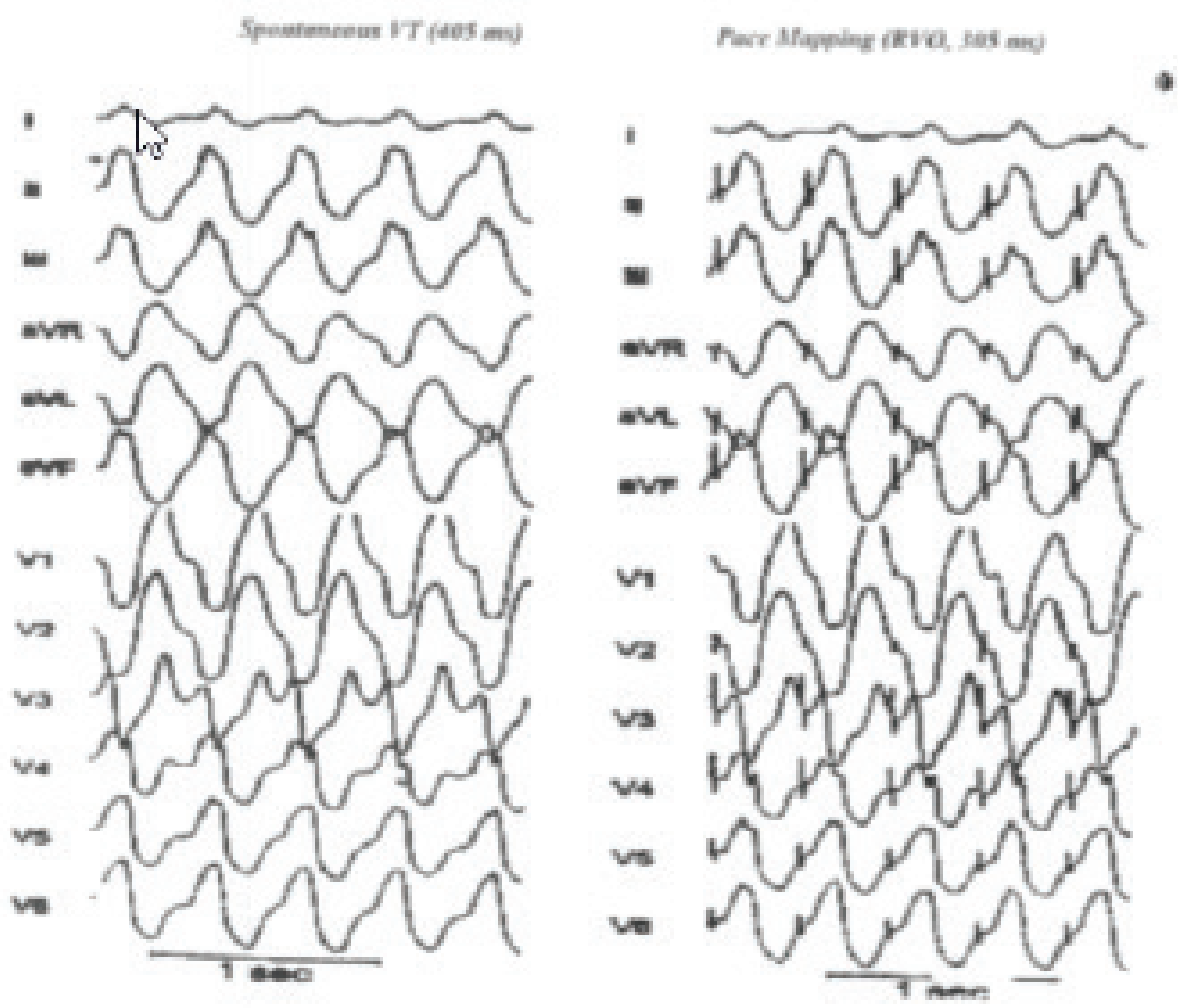

\section{Electrophysiological (EP) features}

Ventricular extra stimulation frequently fails to induce VT. Isoproterenol infusion is often required for mapping. Site of earliest activation during VT is usually septal region of RVOT (preceding the onset of surface QRS by 10-35 ms). Pacing from the site of origin results in an identical ORS morphology as compared with the QRS during spontaneous VT (Fig.1). Autonomic modulation as valsalva, CSM ventricular extra stimulation and overdrive pacing can convert the VT.

Drugs like verapamil, adenosine and b-blockers can also be used to terminate the tachycardia. Idiopathic Right Ventricular Tachycardia is due to triggered activity. Adenosine specifically terminates VT due to triggered activity (DAD). It has no effect on VT due to reentry or abnormal automaticity. 


\section{Treatment and Prognosis}

Drugs like class III, Ic antiarrhythmics, B-blockers and Verapamil can suppress this VT. Radiofrequency catheter ablation effectively eliminates this focal tachycardia in symptomatic patients. Prognosis is favorable in the long run. Recurrence rate is $25-40 \%$ without treatment. Cardiac arrest in the rare cases is reported.

Fig. 2: IDIOPATHIC LEFT VENTRICULAR TACHYCARDIA
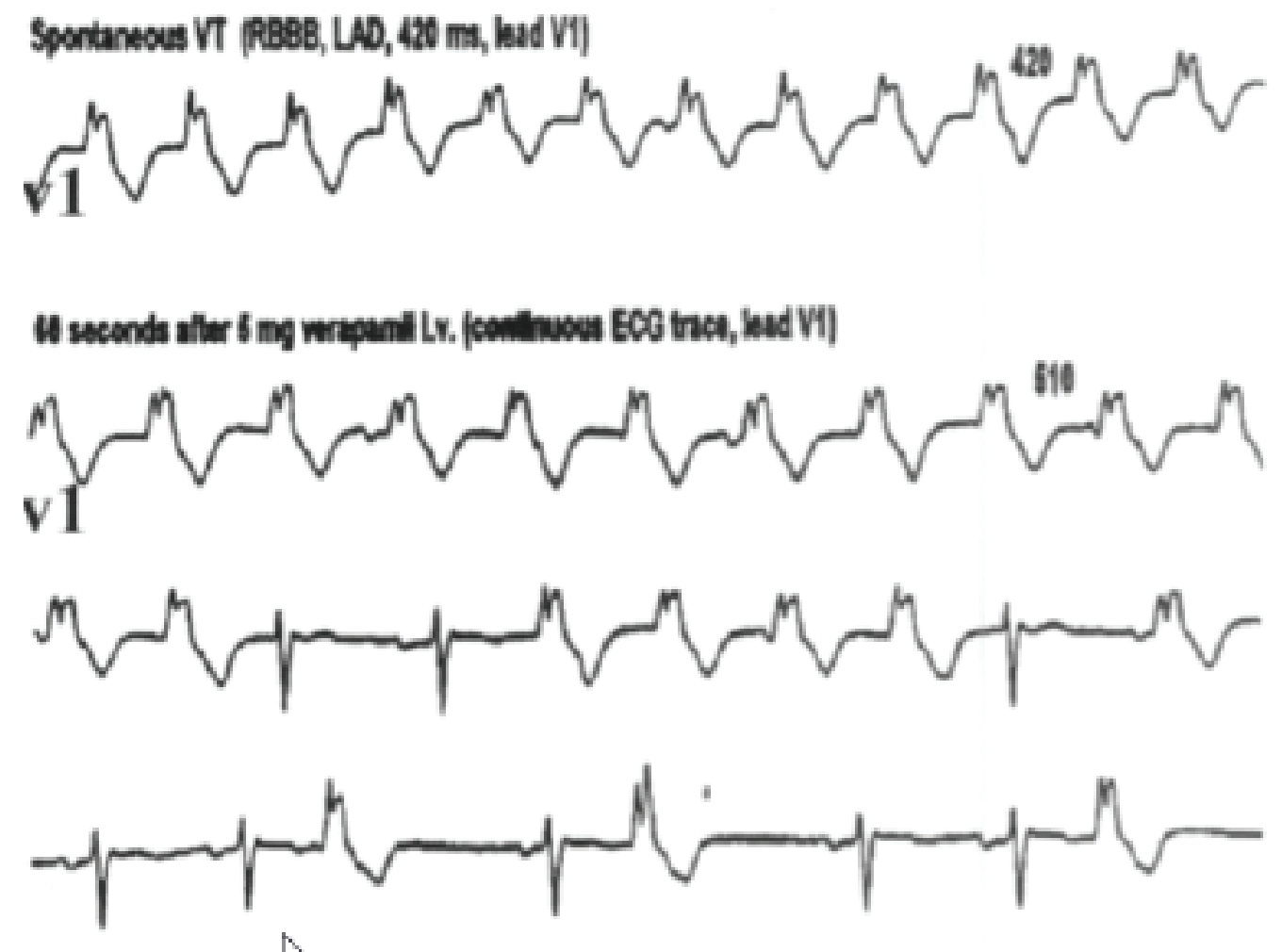

\section{Idiopathic Left ventricular tachycardia}

Response of recurrent sustained VT to verapamil was first reported by Belhassen et al in 1981. The salient features of this VT are RBBB with superior axis suggesting LV origin (Posteroseptal, L fascicle) in the ECG during VT (Fig. 2). Therefore it is often known as "Fascicular Tachycardia". It is usually not related to physical activity, not provokable by exervise testing or isoproterenol infusion. Frequently it is inducible by programmed extra stimulus (POS) using rapid atrial/ventricular pacing or extra stimulation. Typically there is response to Verapamil. Mapping during VT shows site of earliest activation (usually within $20 \mathrm{~ms}$ of QRS onset) in the infero apical/midseptal region of LV. Pacing from the site of the earliest activation produces QRS complexes very similar to those during VT (suggesting the site of VT origin) 


\section{Mechanism}

The most likely mechanism is re-entrant tachycardia and participation of calcium channel mediated pathway within the re-entrant circuit. This is consistent with the positive response to verapamil. B-blockers or lidocaine are not effective here.

\section{Treatment and Prognosis}

Verapamil is the drug of choise. Catheter Ablation effectively eliminates this tachycardia in symptomatic patients. Retrograde aortic or trans-septal approach is used here. InferoPosterior left side of IVS is explored looping the catheter against the lateral wall of LV \& directing the tip towards IVS. The prognosis in this type of VT is favorable. Only in few cases there are development of overt cardiomyopathy or SCD.

\section{Fig. 3: REPETITIVE MONOMORPHIC VENTRICULAR TACHYCARDIA}

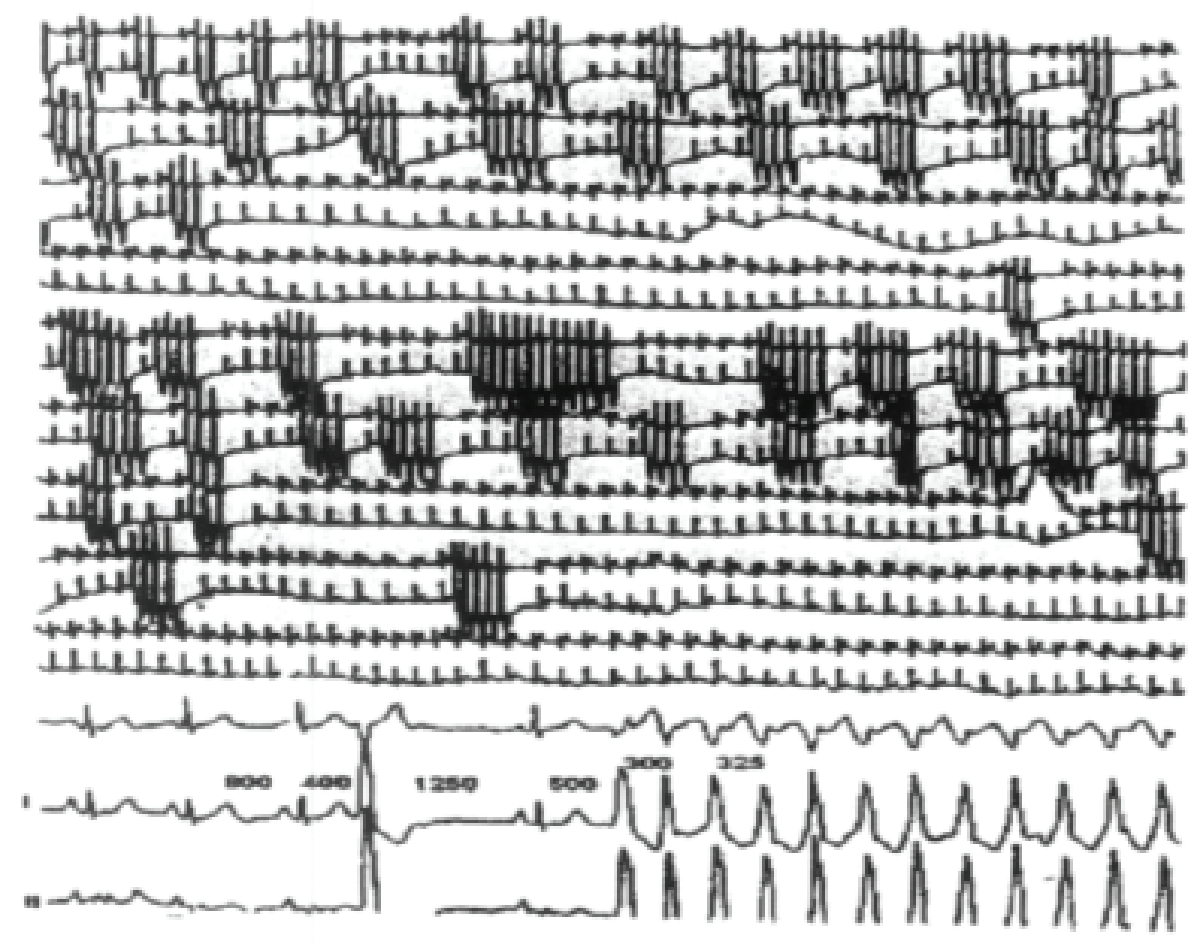

\section{Repetitive Monomorphic VT}

Gallavarian first described this condition as "Extrasystolie Ventricularairea Paroxysmes Tachycardiques Prolonges" in 1922. Extrasystoles and tachycardias are the two components present in this VT. Clinically there are frequent episodes of NSVT interrupted by only short periods of sinus rhythm, more during physical activity (Fig. 3). Mostly it present as LBBB with RAD morphology. It often has critical rate dependant window of sinus rate like VT occurring only if sinus rate falls below 70 or goes above 110 . 


\section{Electrophysiological features}

During EP study it is often not inducible by programmed electrical stimuli (PES) of ventricles. In some atrial pacing can be used along with isoproterenol infusion for induction. Termination or successful ablation is difficult to assess because of the spontaneous termination of VT. Mechanism is possibly triggered activity (DAD).

\section{Treatment and Prognosis}

Drugs like Ic, III antiarrhythmics, B-blockers, Verapamil can suppress this VT. Catheter ablation effectively eliminates this tachycardia in symptomatic patients. Prognosis is favorable but recurrence is common.

\section{Bundle Branch Reentrant Tachycardia}

This is an uncommon type of VT found often with some structural heart disease. H-V interval is prolonged during sinus rhythm. During VT, HV and RB-V intervals are more or same as during sinus rhythm. Presence of fusion complex during atrial pacing excludes the diagnosis.

\section{Mechanism}

Macro reentrant Circuit involving RBB and LBB (Fig. 4). VT usually has LBBB morphology.

Fig. 4: VT INNORMALHEART

SUMMARY

\begin{tabular}{|l|l|l|l|l|}
\hline Mechanism & IRVT & ILVT & RMVT & BBRVT \\
\hline Moctivity & $\begin{array}{l}\text { Triggered } \\
\text { Reentry }\end{array}$ & $\begin{array}{l}\text { Micro- } \\
\text { Activity }\end{array}$ & $\begin{array}{l}\text { Micro- } \\
\text { Reentry }\end{array}$ \\
\hline $\begin{array}{l}\text { Termination } \\
\text { I/V Verapamil } \\
\text { IIV Adenosine }\end{array}$ & + & RBBB/RAD & LBBB/RAD & LBBB \\
\hline $\begin{array}{l}\text { Treatment } \\
\text { D Drugs } \\
\text { RFA }\end{array}$ & $\begin{array}{l}\text { Verapamil, III } \\
++\end{array}$ & $\begin{array}{l}\text { III, Ic. } \\
\text { Verapamil ++ }\end{array}$ & $\begin{array}{l}\text { Variable } \\
++\end{array}$ & $\begin{array}{l}\text { III } \\
++\end{array}$ \\
\hline
\end{tabular}




\section{Treatment}

RF Ablation of RBB is the treatment of choice.

\section{Arrhythmogenic RV Dysplasia and Brugada Syndrome}

Fontaine et al first described ARVD as "A condition presenting as VT with no obvious Heart disease" in 1979. This is a rare, predominantly RV cardiomyopathy found mostly in young/middle aged men. The principle manifestation is VT (LBBB type) and in echo/MRI/ ANGIO reveals ventricular contraction abnormalities. In $50 \%$ patients localized bulges or sacculations are seen. Endomyocardial bipsy (EMB) is usually negative.

BRUGADA first described another peculiar condition presenting as RBBB and persistent ST segment elevation and sudden death as distinct clinical and electrographic syndrome in 1992. However, possibly these two conditions are not mutually exclusive. Tada et al (1998) in fact have shown that right ventricular dysplasia is often present in patients with so called Brugada syndrome.

\section{Bibliography:}

1. Brugada Et Al: RBBB, Persistent ST segment elevation and sudden death: a distinct clinical and electrographic syndrome: JAM CoLLCARDIOL. (1992; 20: 1391-1396)

2. Tada Et Al: "Arrhythmogenic Right Ventricular Dysplasia Underlies the syndrome of RBBB, ST Segment Elevation and Sudden Death." AJC (1998; 81:519-522)

3. Fontaine et al : "A condition presenting as VT with no obvious Heart diseas." (Circulation 1979:59:65)

4. Gallavarian 1. : Extrasystolie Ventricularaire a Paroxysmes Tachycardiques Prologes Arch Mal Coeur (1922; 15:298)

5. Belhassen et al : Idiopathic ventricular tachycardia and fibrillation. (Br. Heart J (1981; 46: 679-682)

6. Katritsis, D. G. et al : Repetitive monomorphic ventricular tachycardia From Cell to Bedside (194,p.900) 
7. Gill, J. S. et al: Efficacy of flecainide, sotalol, and verapamil in the treatment of right ventricular tachycardia in patients without overt cardiac abnormality. Br. Heart J. (68: 292, 1992)

8. Lerman, B. B., et al: Mechanism of repetitive monomorphic ventricular tachycardia. (92: 421,1995)

9. Gill, J. S. et al: Verapamil for the suppression of IVT of LBBB- like morphology. Am. Heart J. 126:1126, 1993

10.Kottkamp, H., et al : Radiofrequency catheter ablation of ILVT: 5: 268, 1994

11. DeLacey, W.A., et al ; Adenosine and verapmil-sensitive ventricular tachycardia originating from the left ventricle: PACE 15: 2240, 1992

* Attending Cardiologist, Escorts Heart Insitute \& Research Centre, New Delhi, India consultant Cardiologist, Norvic Escorts Health Care \& Research Center, Kathmandu, Nepal

${ }^{* *}$ Norvic Escorts Health Care \& Research Center, Kathmandu, constant, UNICEF, Nepal 